\title{
CORRECTION
}

\section{Correction to: Infographic of primary trabeculectomy for advanced glaucoma study (TAGS)}

Clarissa E. H. Fang (D), Rashmi G. Mathew and Christin Henein (D)

(c) The Author(s), under exclusive licence to The Royal College of Ophthalmologists 2021

Eye (2022) 36:1132; https://doi.org/10.1038/s41433-021-01751-3

Correction to: Eye

https://doi.org/10.1038/s41433-021-01701-z.
Unfortunately, the family name by Rashmi G. Mathew was written incorrect.

The original article has been corrected. 